Investigation of Tractor Base Bleeding for Heavy Vehicle Aerodynamic Drag

Reduction

J. Ortega, K. Salari, B. Storms

October 29, 2007

The Aerodynamics of Heavy Vehicles II: Trucks, Buses, and Trains

Tahoe City, CA, United States

August 26, 2007 through August 31, 2007 
This document was prepared as an account of work sponsored by an agency of the United States government. Neither the United States government nor Lawrence Livermore National Security, LLC, nor any of their employees makes any warranty, expressed or implied, or assumes any legal liability or responsibility for the accuracy, completeness, or usefulness of any information, apparatus, product, or process disclosed, or represents that its use would not infringe privately owned rights. Reference herein to any specific commercial product, process, or service by trade name, trademark, manufacturer, or otherwise does not necessarily constitute or imply its endorsement, recommendation, or favoring by the United States government or Lawrence Livermore National Security, LLC. The views and opinions of authors expressed herein do not necessarily state or reflect those of the United States government or Lawrence Livermore National Security, LLC, and shall not be used for advertising or product endorsement purposes. 


\title{
Investigation of Tractor Base Bleeding for Heavy Vehicle Aerodynamic Drag Reduction
}

\author{
Jason Ortega, Kambiz Salari, Bruce Storms
}

\begin{abstract}
One of the main contributors to the aerodynamic drag of a heavy vehicle is tractor-trailer gap drag, which arises when the vehicle operates within a crosswind. Under this operating condition, freestream flow is entrained into the tractor-trailer gap, imparting a momentum exchange to the vehicle and subsequently increasing the aerodynamic drag. While a number of add-on devices, including side extenders, splitter plates, vortex stabilizers, and gap sealers, have been previously tested to alleviate this source of drag, side extenders remain the primary add-on device of choice for reducing tractor-trailer gap drag. However, side extenders are not without maintenance and operational issues. When a heavy vehicle pivots sharply with respect to the trailer, as can occur during loading or unloading operations, the side extenders can become crushed against the trailer. Consequently, fleet operators are forced to incur additional costs to cover the repair or replacement of the damaged side extenders. This issue can be overcome by either shortening the side extenders or by devising an alternative drag reduction concept that can perform just as effectively as side extenders. To explore such a concept, we investigate tractor base bleeding as a means of reducing gap drag. Wind tunnel measurements are made on a 1:20 scale heavy vehicle model at a vehicle width-based Reynolds number of 420,000 . The tractor bleeding flow, which is delivered through a porous material embedded within the tractor base, is introduced into the tractor-trailer gap at bleeding coefficients ranging from 0.0-0.018. To determine the performance of tractor base bleeding under more realistic operating conditions, computational fluid dy-
\end{abstract}

Jason Ortega

Lawrence Livermore National Laboratory, P.O. Box 808, L-644, Livermore, CA 94550, e-mail: ortega17@1lnl.gov

Kambiz Salari

Lawrence Livermore National Laboratory, P.O. Box 808, L-644, Livermore, CA 94550, e-mail: salari1@1lnl.gov

Bruce Storms

NASA Ames Research Center, Moffett Field, MS-260, CA 94035, e-mail: bstorms@mail.arc.nasa.gov 
namics simulations are performed on a full-scale heavy vehicle within a crosswind for bleeding coefficients ranging from $0.0-0.13$.

\section{Introduction}

During the 1970s and 1980s, a number of first-generation drag reduction devices were designed to reduce the aerodynamic losses of heavy vehicles [5]. The result of this effort led to the development of a number of devices (front-end rounding, tractor aero-shields, body molding, and cabin side extenders) that improved the aerodynamics of heavy vehicle tractors. Additionally, a number of second-generation devices (tractor-trailer gap sealers, trailer side skirts, and boattails) were developed. Unfortunately, these devices did not enter into the market on a wide-scale basis, which was due not to their ineffectiveness in reducing drag, but rather to operational, maintenance, and, ultimately, economic concerns. However, with rising fuel costs and potentially unstable fuel supplies, there is a renewed objective to further reduce heavy vehicle aerodynamic drag.

On a modern heavy vehicle, one of the main sources of aerodynamic drag is tractor-trailer gap drag, which occurs when the vehicle is operating in a crosswind and free-stream flow is entrained into the tractor-trailer gap. This flow entrainment imparts a momentum exchange to the heavy vehicle in the direction opposite of travel, resulting in a drag increase. Presently, cabin side extenders, which are $0.46-0.51 \mathrm{~m}$ wide vertical plates attached to the tractor base, are routinely utilized on modern heavy vehicles to mitigate flow entrainment into the gap. Wind tunnel measurements have shown that side extenders, when used in conjunction with a rooftop aero-shield and tractor side skirts, reduce the wind-averaged drag coefficient, $C_{d w a v g}$, by about 0.15 [6]. Other devices, such as gap sealers and fillers are also effective in reducing tractor-trailer gap drag and provide additional reductions in the wind-averaged drag coefficient ranging from about 0.03-0.06 [6]. However, due to maintenance and operational issues, these devices are not commonly installed on modern heavy vehicles.

Despite their widespread use, side extenders are often damaged during routine shipping operations. Commercial fleet owners frequently comment that the large structural surfaces comprising the side extenders are easily bent or crushed when the tractor pivots too sharply relative to the trailer during loading maneuvers. When such damage occurs, the commercial fleets are forced to take the tractor out of operation for service and either remove or replace the side extenders, a task which can become extremely costly when such repairs are required for several thousand tractors in the larger shipping fleets. Consequently, there is a need to devise alternate drag reduction concepts that can both alleviate flow entrainment into the tractor-trailer gap and be less prone to damage during vehicle articulation. One such concept recently developed are side extenders that are about $38 \%$ shorter than those presently used and still provide nearly the same aerodynamic benefit [7]. 
Another proposed concept is tractor base bleed, in which a stream of low-speed flow is injected into the tractor-trailer gap at a velocity, $U_{b}$, over a large surface area, $A_{b}$, on the tractor base. Base bleed has been previously studied as a means of reducing the drag of bluff bodies, such as ballistic shells [18], blunt-base airfoils [28], backward facing steps [16], and circular cylinders [23]. The typical values of bleeding flow, defined by the bleeding coefficient, $C_{\mu}=U_{b} A_{b} / U_{\circ} A_{\circ}$, range from 0.0-0.28, where $U_{\circ}$ is the free-stream velocity and $A_{\circ}$ the characteristic area of the body. Bearman [3], Michel \& Kost [20], and Wood [28, 29] showed that base bleed increases the pressure over the bleeding surface, thereby reducing the overall drag of the bluff body. When sufficient bleeding flow is injected into the separated wake, Bearman [3], Schumm, et al. [23], and Wood [28, 29] demonstrated that the strength of shed vortex street can be reduced. In addition, base bleeding displaces the separated wake in the downstream direction [3, 24, 29, 30]. Further increases in bleeding can suppress vortex shedding entirely. Yao \& Sandham [30] showed that bleeding over a large area at a low velocity is more effective in reducing the drag than bleeding over a small area at a high velocity. Base bleed has also been shown to influence the stability characteristics of the separated wake [11]. Arcas \& Redekopp [1], Hammond \& Redekopp [10], Huerre \& Monkewitz [11], and Sevilla \& Martinez-Bazan [24] observed that, at a critical bleed rate, the wake transitions from being absolutely unstable to convectively unstable. And, Koch $[14,15]$ showed that the transition from absolute to convectively unstable flow occurs slightly downstream of the region of reverse flow in the separated wake.

Although the previous studies elucidated the flow physics and drag reduction capability of base bleeding, the types of bluff bodies used were either two-dimensional or axisymmetric. In addition, the bluff bodies were isolated from the influence of other bodies and were oriented at zero degrees yaw with respect to the freestream flow. It is therefore difficult to determine from these previously published results as to whether or not base bleeding is an effective means of reducing the drag of a tractor-trailer gap, which is surrounded by both a highly asymmetric, threedimensional geometry and a moving ground plane and which is typically oriented at a finite yaw angle. Thus, the purpose of this study is to investigate base bleeding as means of reducing tractor-trailer gap drag. In particular, we wish to answer the following questions. Does tractor base bleeding reduce the drag of a heavy vehicle? How much bleeding flow is required to produce a modest drag reduction? How is the flow within the tractor-trailer gap altered as a result of base bleeding? How do the gap flow physics and the reductions, if any, in drag due to tractor base bleeding compare to those of traditional side extenders?

\section{Wind Tunnel Measurements}

As a first step in providing answers to these questions, we perform wind tunnel measurements on a 1:20 scale heavy vehicle model that employs tractor base bleeding. The model tractor, which is a detailed representation [27] of a Freightliner 
Columbia [8], is fabricated using a rapid prototyping technique (Fig. 1a) [25]. The engine grill and the air inlets on the bumper are sealed since Leuschen \& Cooper [17] measured very little difference in the drag coefficient when the cooling airflow through these two regions was blocked. To prevent laminar flow separation, several lengths of $4 \times 10^{-4} \mathrm{~m}$ diameter wire are laid over the leading edges of the tractor and $4.6 \times 10^{-4} \mathrm{~m}$ diameter glass beads are bonded to the tractor nose, visor, aeroshield, window pillars, door columns, and engine grill. The tractor is attached to a $0.69 \mathrm{~m}$ long model freight van that includes cross members and a landing gear on the trailer underside. The trailer body is constructed from $0.006 \mathrm{~m}$ thick sheets of acrylic, while the trailer wheels are fabricated in the same manner as that of the tractor. The tractor-trailer gap is adjustable and, for this study, is set to 0.038 and $0.076 \mathrm{~m}$. Force measurements are made by suspending the entire tractor-trailer model on a six-component force balance (Northrop MK XVA 0.75") that is positioned near the upstream end of the trailer. The signals from the force balance are digitized and stored on a computer using BDAS 9.3 data acquisition software [4]. The resulting drag coefficients, $C_{d}=D / \frac{1}{2} \rho U_{\circ}^{2} A_{\circ}$, are obtained to within $\pm 1.69 \times 10^{-3}$, which includes both the balance accuracy and the average measurement repeatability, where $D$ is the drag force along the body axis, $x_{a}$, of the model, $\rho$ the density of air, and $A_{\circ}=2.47 \times 10^{-2} \mathrm{~m}^{2}$ the characteristic area of the model.

Tractor base bleeding is provided by directing a compressed air supply into two $0.019 \mathrm{~m}$ NPT air supply lines that are connected to two smaller $0.006 \mathrm{~m}$ internal diameter tubes, which enter the model through the trailer underside (Fig. 1b). The bleeding flowrate is measured with a pneumatic in-line flowmeter (Omega FL7722A). Since the $0.006 \mathrm{~m}$ internal diameter tubes pass from the non-metric to the metric portions of the force balance, a service loop is incorporated into the tubing before it exits the upstream end of the trailer underside and is attached to two ports on the tractor underside. These ports direct the airflow into a plenum that comprises the entire model tractor cabin. On the base of the tractor cabin are threaded holes to which several different rapid-prototyped pieces can be affixed. For base bleeding configurations, perforated tractor bases with and without $0.02 \mathrm{~m}$ wide side extenders are employed. On these pieces, the bleeding surface area is about $19 \%$ of the characteristic area, $A_{\circ}$, of the model tractor. For cases in which there is no bleeding flow, aluminum duct tape is placed over the perforated holes or a separate non-perforated tractor base piece with or without side extenders is fixed to the tractor base. Within the plenum, $0.051 \mathrm{~m}$ thick packing foam is positioned against the perforated tractor base to provide a pressure drop for the bleeding flow as it exits the plenum, thereby ensuring a more uniform bleeding velocity profile. Four bleeding flowrates, corresponding to base bleeding coefficients of $0.0,0.008,0.013$, and 0.018 , are used in this wind tunnel study. Velocity measurements made in the gap using a multi-hole pressure probe (Cobra, Turbulent Flow Instrumentation) with no tunnel velocity demonstrate fairly uniform bleeding velocity (Fig. 2).

The drag coefficient measurements are made within the NASA Ames Fluid Mechanics Laboratory open-circuit wind tunnel, which has a contraction ratio of 9:1, a test section size of $0.813 \mathrm{~m} \times 1.219 \mathrm{~m}$, and a free-stream turbulence level of $0.15 \%$. The wind tunnel is operated at a nominal dynamic pressure and tunnel speed of 
a)

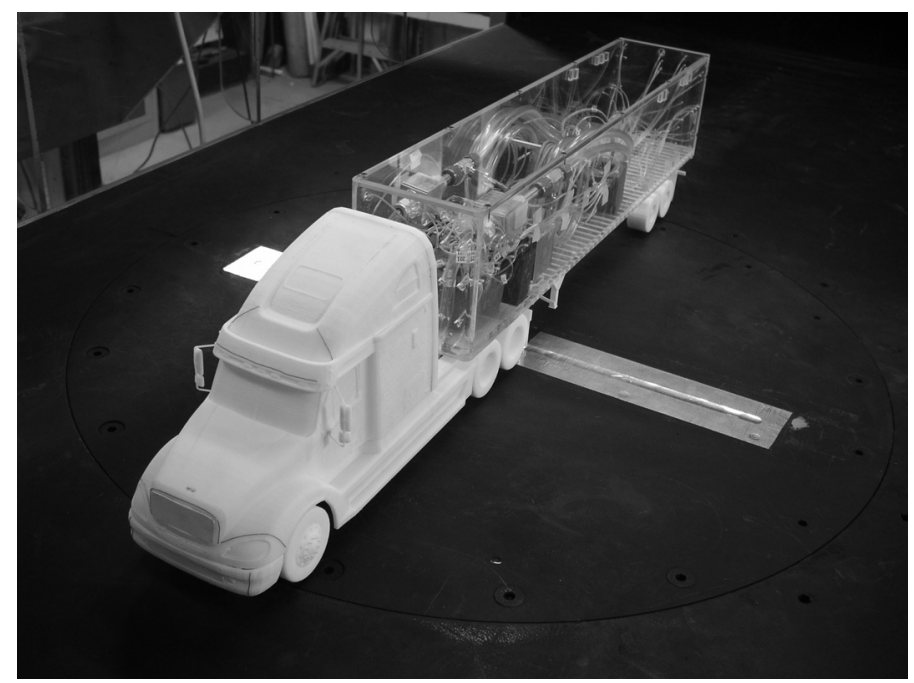

b)

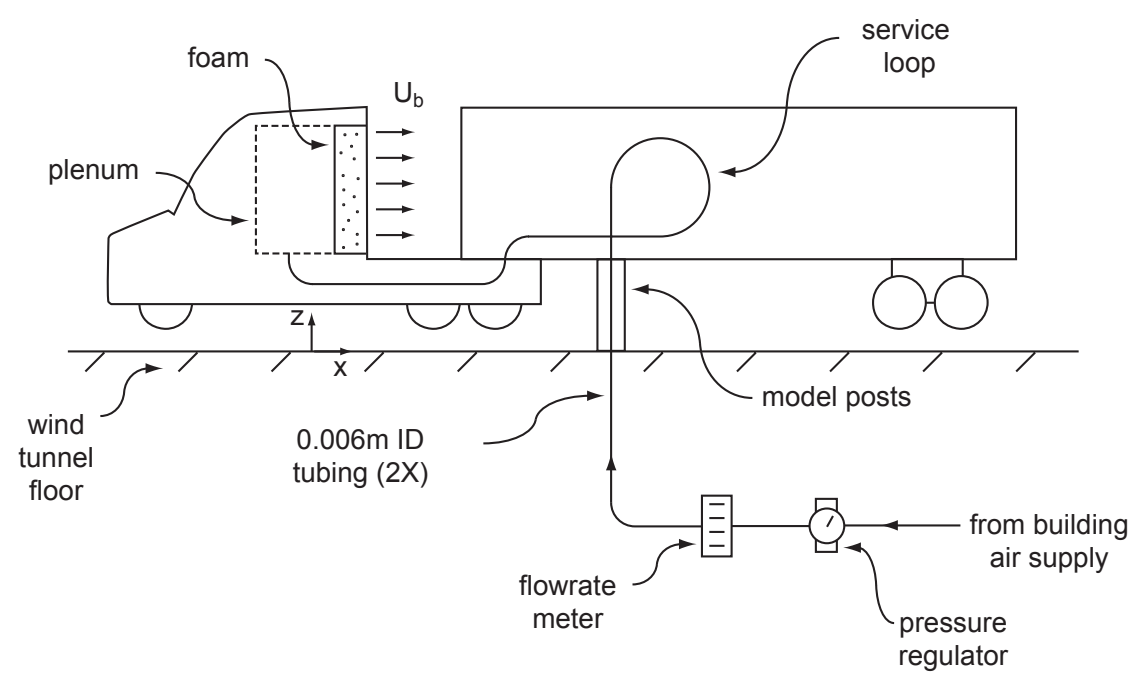

Fig. 1 a) 1:20 scale heavy vehicle model model in the low-speed NASA Ames wind tunnel. b) Details of the tractor base bleeding system. 


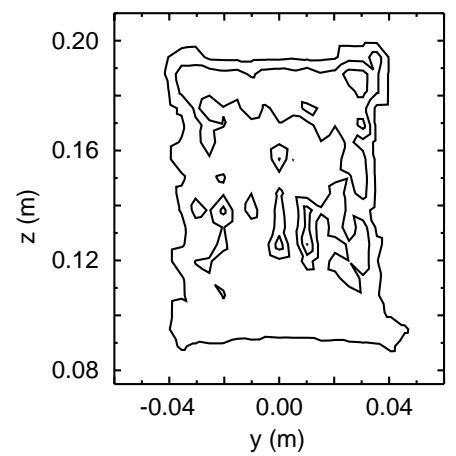

a)

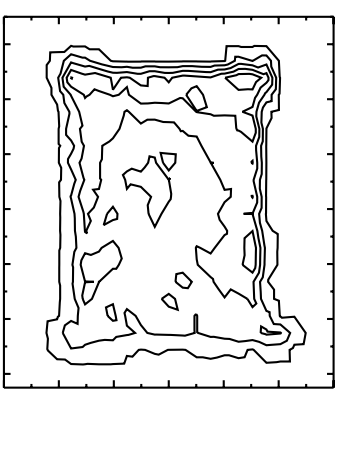

b)

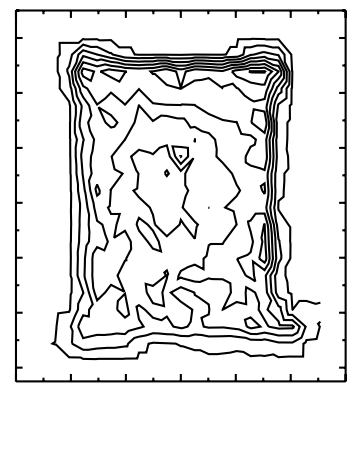

c)

Fig. 2 Bleeding velocity contours in the $x$-direction made $0.019 \mathrm{~m}$ downstream of the tractor base for $C_{\mu}$ equal to a) 0.008, b) 0.013 , and c) 0.018 for a tractor-trailer gap distance of $0.076 \mathrm{~m}$ with no tunnel velocity.

$1420 \mathrm{~N} / \mathrm{m}^{2}$ and $48.95 \mathrm{~m} / \mathrm{s}$, respectively. The resulting width-based Reynolds number, $R e_{w}=U_{\circ} w / v$, of the model is 420,000 , where $w=0.13 \mathrm{~m}$ is the model width, and $v$ the kinematic viscosity of air. The percentage of blockage of the model in the tunnel test section is $2.5 \%$ at $0^{\circ}$ yaw. The model is mounted at height about equal to the boundary layer displacement thickness $\left(\approx 5.0 \times 10^{-3} \mathrm{~m}\right)$ above the tunnel floor on a turntable that is driven by a computer-controlled servo motor (Parker Compumotor), which can rotate the model to within $\pm 0.05^{\circ}$. For each model configuration, force measurements are made at yaw angles ranging from $\pm 8^{\circ}$. To assess the repeatability of the measurements, as well as hysteresis in the flow patterns over the model, the data are acquired on selective configurations for both increasing and decreasing yaw angle directions and are observed to show negligible hysteresis effects. Since the tractor bleeding flow generates forces on the model due to the effects of thrust and the expansion of the $0.006 \mathrm{~m}$ internal diameter tubing within the trailer, force measurements are first made at a zero tunnel velocity at each non-zero bleeding coefficient value. These forces are then subtracted from the subsequent measurements made at the corresponding bleeding coefficient values.

While the $C_{d}$ data at each yaw angle is useful for evaluating the tractor base bleeding concept, it is somewhat cumbersome since the $C_{d}$ distribution does not summarize the drag reduction performance into a single quantity that can be easily compared with that of other devices, such as side extenders. Taking the mean value of $C_{d}$ over all measured yaw angles is also insufficient since it does not account for the fact that the crosswind velocities cause a vehicle traveling at a particular speed to experience certain yaw angles more than others. A quantity that resolves both of these issues is the wind-averaged drag coefficient, $C_{d w a v g}$ [12], which is computed for each base bleeding configuration (Fig. 3). It is apparent that the drag coefficient decreases as the bleeding coefficient is increased for both gap distances. However, a greater reduction in drag is observed for the larger gap distance both with and without side extenders. When $C_{\mu}>0.006$, tractor base bleeding with no 
side extenders at the larger gap distance yields a greater reduction in drag than that of side extenders alone with no bleeding flow. It should also be noted that the windaveraged drag coefficients for the larger gap are greater than those of the smaller gap, indicating the presence of a larger amount of entrained flow into the tractor-trailer gap. Similar trends in the dependence of the drag coefficient upon gap distance, $d_{\text {gap }}$, were observed by Hammache \& Browand [9], who investigated tractor-trailer gap flow dynamics on a simplified tractor-trailer geometry at a comparable $R e_{w}$ of 270,000. At a critical gap distance of $d_{\text {gap }} / \sqrt{A_{\circ}} \approx 0.5$, Hammache \& Browand showed that the total vehicle drag increases sharply. In the present study, the values of $d_{g a p}=0.038$ and $0.076 \mathrm{~m}$ are below and about equal to the critical gap distance, respectively.

Fig. 3 Wind-averaged drag coefficient, $C_{d w a v g}$, as a function of the tractor base bleeding coefficient, $C_{\mu}$, for two tractor-trailer gap distances with and without side extenders.

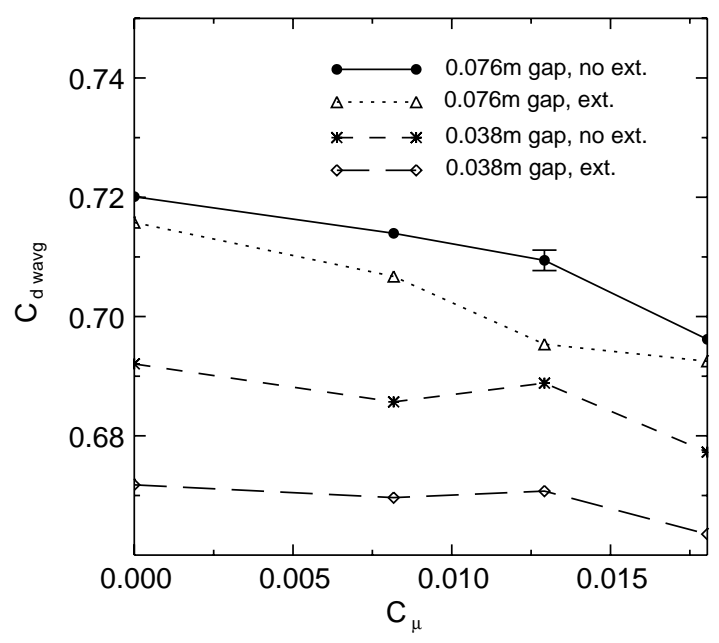

\section{CFD Simulations}

While the wind tunnel measurements demonstrate that tractor base bleeding can indeed reduce the drag of the heavy vehicle model, further investigation is needed both to demonstrate the effectiveness of this concept at a full-scale Reynolds number and to understand the changes in the gap flow physics arising from the base bleeding. For these reasons, we perform computational fluid dynamics (CFD) simulations on a full-scale Freightliner Columbia operating at highway conditions within a crosswind. The tractor-trailer gap distance is set to $1.02 \mathrm{~m}$ and a $13.6 \mathrm{~m}$ long freight van is attached to the tractor.

The simulations are performed within a computational domain that is $98 \mathrm{~m} \times$ $49 \mathrm{~m} \times 128 \mathrm{~m}$ (Fig. 4), such that the heavy-vehicle cross-sectional area is $0.2 \%$ of that of the domain. To model the crosswind velocity, $U_{w}$, which is typically $3.1 \mathrm{~m} / \mathrm{s}$ 
at vehicle mid-height [22], while the vehicle travels at a ground speed of $U_{g}=$ $U_{\circ}=29.1 \mathrm{~m} / \mathrm{s}$, the vehicle is yawed to $\theta=\tan ^{-1}\left(U_{w} / U_{g}\right)=6.1^{\circ}$ and a velocity of $\sqrt{U_{g}^{2}+U_{w}^{2}}=29.3 \mathrm{~m} / \mathrm{s}$ is specified at the inlet to the computational domain (Fig. 4). The resulting width-based Reynolds number is 5,000,000. Beneath the vehicle, a no-slip, moving ground plane boundary condition is prescribed at a velocity of $U_{g}$ and a yaw angle of $6.1^{\circ}$. The no-slip surfaces of the tractor and trailer tires, which rotate at an angular velocity of $53 \mathrm{~s}^{-1}$, intersect the ground plane, producing a tire contact patch that has a swept angle of $20^{\circ}$ [2]. A slip boundary condition and a zero gradient boundary condition are specified along the walls and outlet, respectively, of the computational domain. To produce the tractor base bleeding flow, an inlet velocity boundary condition $\left(U_{b}=0.0-0.2 U_{\circ}, v_{c}=0, w_{c}=0\right)$ is defined across the tractor base, which has an area that is $65 \%$ of that of the vehicle cross-section, $A_{o}=$ $9.87 \mathrm{~m}^{2}$. The corresponding bleeding coefficients range from $0.0-0.13$. The other variables on the bleeding inlet boundary are computed by assuming a zero gradient normal to the inlet, such that the variables on the inlet nodes are extrapolated from the values of the interior nodes. To provide a performance comparison for the base bleeding concept, side extenders, which have a length of $0.38 \mathrm{~m}$, are modeled in one vehicle configuration and compared with the baseline case. A finite-volume code [26] is used to solve the Reynolds averaged Navier-Stokes (RANS) equations for the flow about the heavy vehicle. The spatial fluxes are discretized with a secondorder monotone advection and reconstruction scheme (MARS) [26] and the $k-\omega$ SST turbulence model [19] with a wall function is used to solve for the turbulent kinetic energy and dissipation fields. A previous validation study by Pointer [21] demonstrated that this turbulence model can adequately capture the value of $C_{d}$ at a $R e_{w}$ on the order of $10^{6}$. The cell height adjacent to the vehicle is chosen to be $3.3 \times 10^{-3} \mathrm{~m}$, yielding a nominal value for $\mathrm{y}^{+}[13]$ of 90 over the entire tractor-trailer surface. Meshes with about $25 \times 10^{6}$ cells are used in this study. The simulations are typically run for about $10,000-20,000$ iterations in order to obtain statistically converged average drag coefficients, which tend to oscillate by about \pm 0.007 due to the inherent unsteady nature of the three-dimensional flow field about the heavy vehicle.

Fig. 4 Computational domain used for the full-scale CFD simulations.

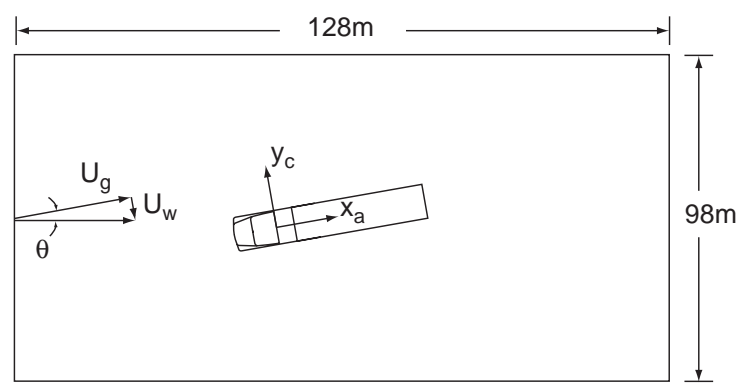


Fig. 5 Change in the drag coefficient as function of the bleeding coefficient for the CFD simulations.

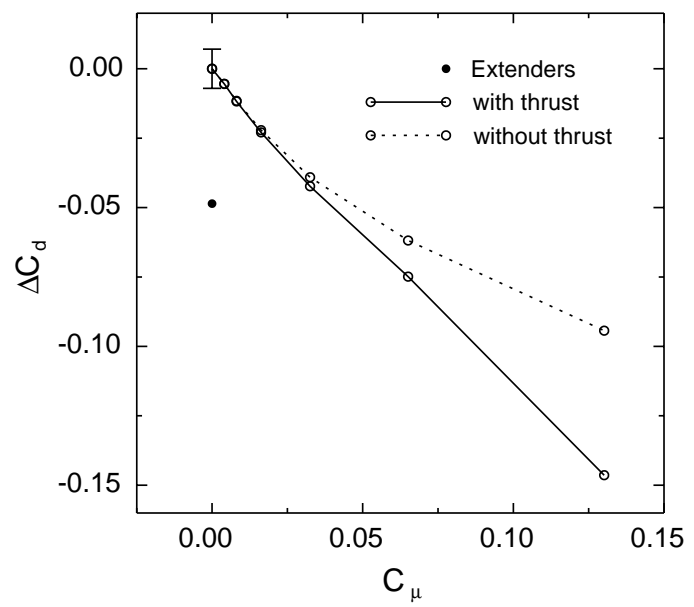

A plot of the drag coefficient as function of the bleeding coefficient (Fig. 5) reveals that, in much the same manner as that of the wind tunnel measurements, increases in bleeding flow decrease the drag coefficient. In addition, the drag reduction of $\Delta C_{d} \approx 0.025$ observed for $C_{\mu} \approx 0.018$ is about equal to that seen experimentally $\left(\Delta C_{d} \approx 0.02\right)$ at $6^{\circ}$ yaw for the larger gap distance despite the relatively large difference in $R e_{w}$. For the computational results, the drag coefficient decreases in a nearly linear fashion for the largest bleeding coefficients, values of which were not attainable in the wind tunnel measurements. The largest reduction in $\operatorname{drag}\left(\Delta C_{d}=\right.$ 0.146 ) occurs at the maximum bleeding coefficient of 0.13 . It is also evident bleeding coefficients greater than 0.06 provide a larger reduction in drag than that of the side extenders $\left(\Delta C_{d}=0.049\right)$. Obviously, a portion of the drag reduction produced by base bleeding is due to the thrust,

$$
T=\int_{A_{b}} \rho U_{b} \mathbf{u} \cdot \mathbf{n} d A
$$

of the bleeding flow and not to modifications of the tractor-trailer gap flow physics, where $\mathbf{u}$ is the fluid velocity vector and $\mathbf{n}$ the unit normal vector of the vehicle surface. Removing this thrust contribution from the data in Fig. 5 demonstrates that, for the bleeding coefficients evaluated in this study, a significant drag reduction occurs solely due to changes in the gap flow physics.

One of the changes to occur is in the amount of free-stream fluid entrained into the tractor-trailer gap. The entrainment rate is computed by defining a rectangular control surface that encompasses the volume of the entire tractor-trailer gap (Fig. 6), but not the bleeding area over the tractor base. The entrainment flowrate,

$$
Q_{e}=\int_{A_{i n}} \mathbf{u} \cdot \mathbf{n}_{c s} d A
$$

is calculated over the control surface to determine the amount of fluid entering the gap from the free-stream, where $\mathbf{n}_{c s}$ is the normal vector of the control surface and 
the integration area, $A_{i n}$, indicates that the integral is computed over those portions of the control surface in which $\mathbf{u} \cdot \mathbf{n}_{c s}<0$. Without any base bleeding, the gap entrains free-stream fluid at a rate of $0.085 U_{\circ} A_{\circ}$, where a large portion of the entrainment occurs on the windward-downstream and bottom-upstream areas (Fig. 6) of the control surface. When side extenders are placed on the tractor base, $Q_{e}=0.072 U_{\mathrm{\circ}} A_{\circ}$. Due to the highly three-dimensional nature of the velocity field in the gap, the flow exits through distinct patches spread over the leeward and upper areas of the control surface. When $C_{\mu}>0$, the bleeding flow reduces the amount of free-stream fluid entrained into the tractor-trailer gap (Fig. 7). (Note that the asymptotic decay of $Q_{e}$ to what appears to be a value greater than 0 is a result of the manner in which the control surface geometry is defined. The value of $Q_{e}$ will likely be greater than zero for all $C_{\mu}$ because free-stream fluid will continue to enter the control surface through the upstream, vertical face.) A comparison of the contour plots of $\mathbf{u} \cdot \mathbf{n}_{c s}$ (Fig. 8) reveals that base bleeding significantly reduces the entrainment of free-stream fluid on the windward and bottom areas of the control surface and produces an exit flow pattern that is more uniformly distributed over the downstream areas of the control surface.

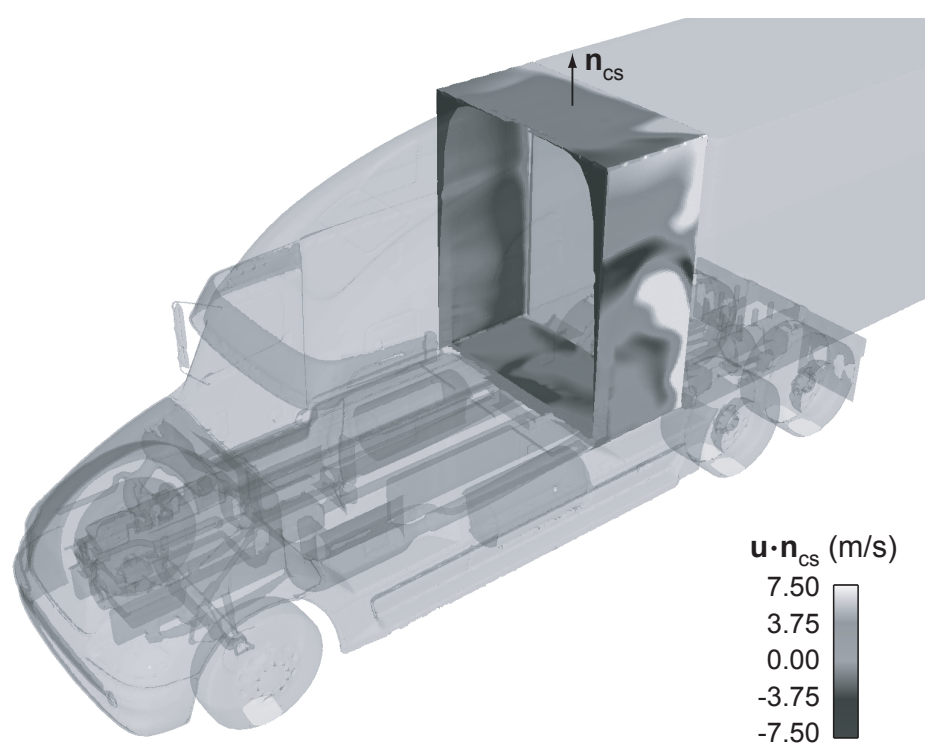

Fig. 6 Control surface defined for computing the flow entrainment rate, $Q_{e}$, into the tractor-trailer gap. The color contours on the surface denote the values of $\mathbf{u} \cdot \mathbf{n}{ }_{c s}$ for $C_{\mu}=0.0$.

The specific manner in which fluid entrainment is altered as a result of base bleeding can be understood by investigating the velocity field within the tractortrailer gap. Prior to base bleeding, the windward shear layer separating from the tractor base impinges upon the trailer frontal face, producing a cross-stream flow in 
Fig. 7 Non-dimensional flow entrainment rate into the tractor-trailer gap as function of the bleeding coefficient. The symbol $\bullet$ denotes the configuration with side extenders $\left(C_{\mu}=0.0\right)$.

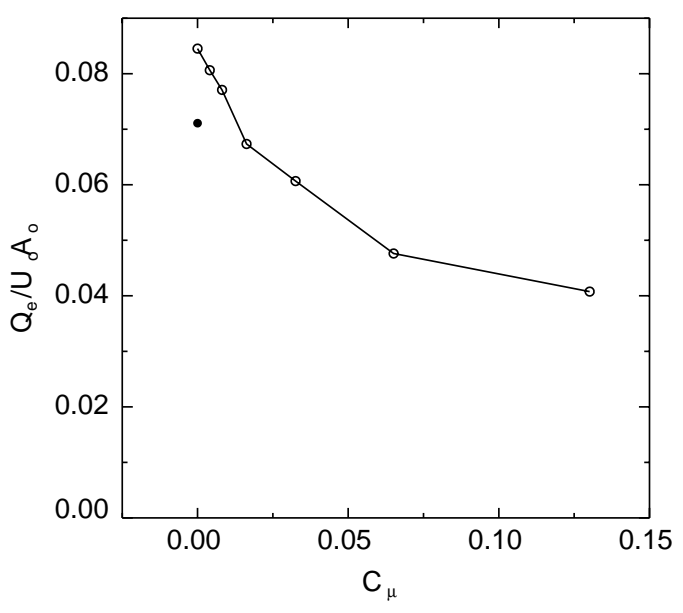

the form of a wall jet that spans the entire gap width (Fig. 9a,c). At the centerline of the gap, this wall jet has a maximum magnitude that is about $0.75 U_{\text {。 }}$ (Fig. 10). Since the flow recirculates within the gap, a positive cross-stream velocity is present near the tractor base. As the amount of base bleeding is increased, the strength of the wall jet decreases. In addition, the cross-stream velocity profile becomes linear with the gap distance near the tractor base and the peak velocity of the positive-valued recirculating flow diminishes. At a bleeding coefficient of $C_{\mu}=0.13$, the crossstream velocity profile is linear throughout the entire tractor-trailer gap centerline, except within a thin boundary layer on the trailer frontal surface. Plots of the velocity field (Fig. 9b,d) show a relatively uniform gap flow, where the regions of large crossstream flow are confined to the outer extents of the gap. It should also be noted that side extenders reduce the strength of the cross-stream flow, such that the maximum magnitude of the wall-jet is reduced to a value about equal to that which occurs when $C_{\mu} \approx 0.033$ (Fig. 10).

The shear layer that produces the wall jet within the gap leaves a distinct high pressure signature as it impinges upon the trailer frontal face (Fig. 11a). Away from this impingement zone, the pressure is considerably lower in magnitude, except for a region on the leeward side of the gap. When bleeding flow $\left(C_{\mu}=0.13\right)$ is applied to the tractor base, this high pressure signature is diminished (Fig. 11b) and replaced with a more uniform pressure distribution that extends over nearly the entire trailer frontal face. The application of side extenders also reduces the pressure signature from the windward shear layer (Fig. 11c), though not to the extent seen for $C_{\mu}=0.13$.

Plots of the pressure coefficient acting in the body axis-direction, $C_{p a}=\mid \mathbf{n}$. $\mathbf{n}_{a} \mid\left(P-P_{\circ}\right) / \frac{1}{2} \rho U_{\circ}^{2}$, (Fig. 12) along the vertical centerline of the tractor base and trailer frontal face demonstrate that the pressure increases on both surfaces as the bleeding coefficient is increased, where $P$ is the fluid pressure, $P_{\circ}$ the freestream pressure, and $\mathbf{n}_{a}$ is the unit body-axis direction vector. Calculating the average value 

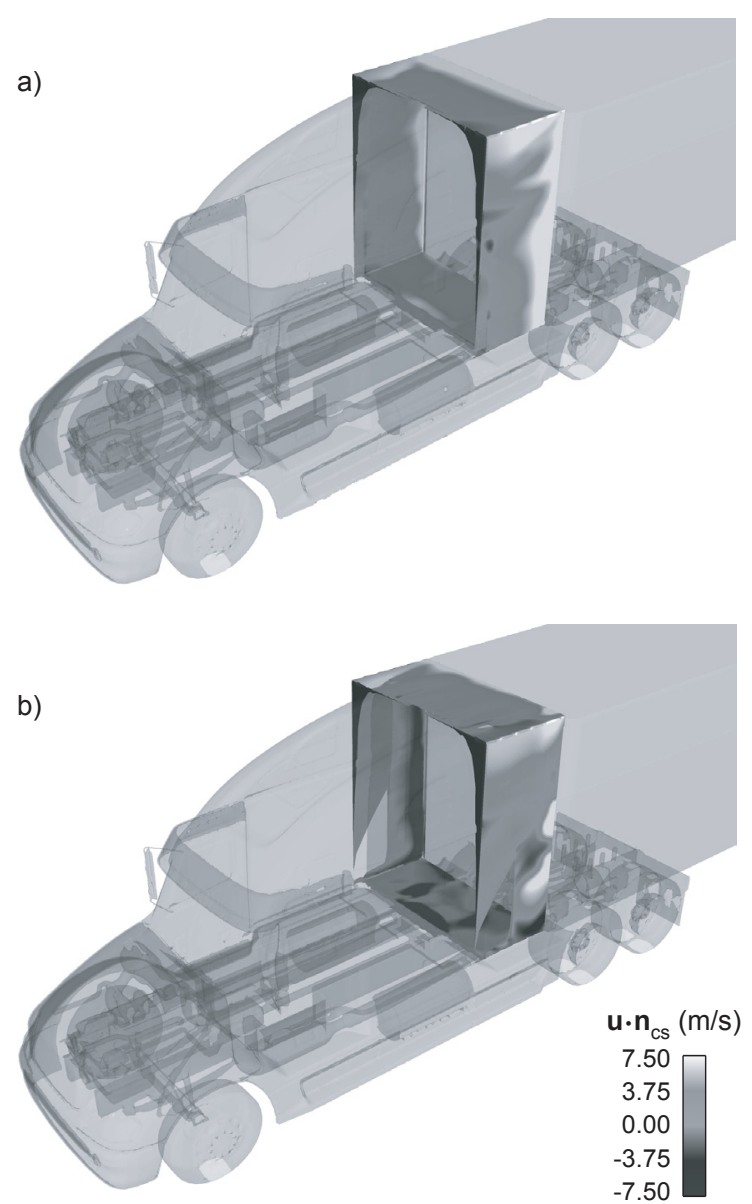

Fig. 8 Contours of $\mathbf{u} \cdot \mathbf{n}_{c s}$ over the control surface for a) a bleeding coefficient of $C \mu=0.13$ and b) for side extenders $\left(C_{\mu}=0.0\right)$.

of $C_{p a}$ over the tractor base, trailer frontal face, and trailer base shows that the average pressure coefficient acting in the body-axis direction on the tractor base increases more rapidly than that on the trailer frontal face as $C_{\mu}$ is increased (Fig. 13). This subsequently reduces the difference between the average pressure coefficients on the tractor base and trailer frontal face, which is, in turn, responsible for the reduction in drag on the entire vehicle. In fact, for $C_{\mu}>0.053$, the average pressure coefficient on the tractor base is greater than that of the trailer frontal face. The side extenders reduce the difference between average pressure coefficients over the tractor base and trailer frontal face, as well. However, the average pressure coefficient on the trailer base exhibits little sensitivity to either the bleeding flow or the side extenders. 
a)

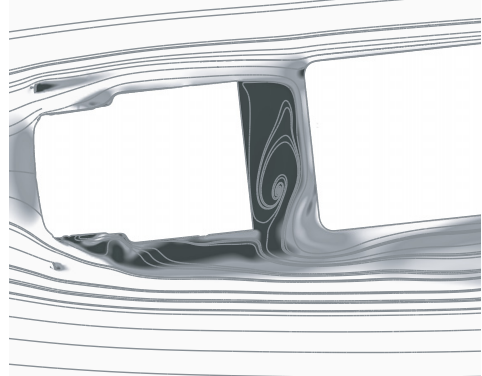

b)

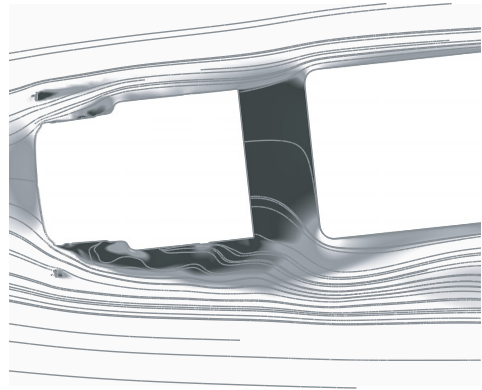

$|\mathbf{u}|(\mathrm{m} / \mathrm{s})$

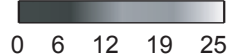

c)

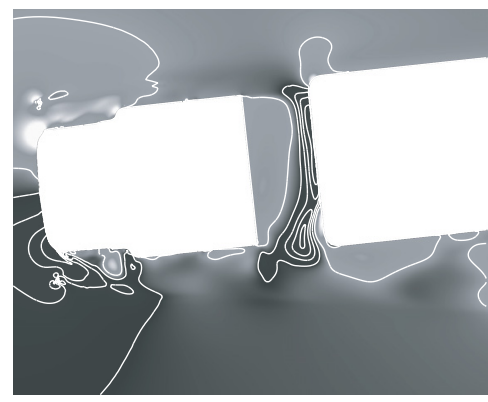

d)

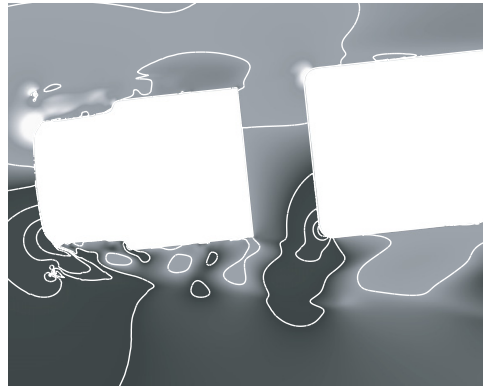

$v_{c x y}(m / s)$

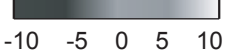

Fig. 9 a-b) Velocity magnitude and streamlines and c-d) cross-stream velocity at the mid-height of the tractor-trailergap for $C_{\mu}$ equal to a,c) 0.0 and b,d) 0.13 . Note that additional negative-valued contours are applied in c-d) to highlight the cross-stream gap flow.

\section{Conclusions}

Through this investigation, we have studied the drag reduction capability of tractor base bleeding. Both the 1:20 scale wind tunnel measurements and full-scale CFD simulations demonstrate that tractor base bleeding reduces the drag coefficient of a heavy vehicle. For bleeding coefficients greater than 0.06 , the simulations show a reduction in the drag coefficient that is greater than that of side extenders. The simulation results also highlight the flow physics within the tractor-trailer gap that contribute to this drag reduction. As the bleeding coefficient increases, the rate of fluid entrainment from the free-stream into the gap decreases, which reduces both the pressure signature of the impinging windward shear layer on the trailer frontal face and the strength of the cross-stream wall jet within the gap. Lastly, tractor base bleeding reduces the difference between the average pressure coefficients on the 
a)

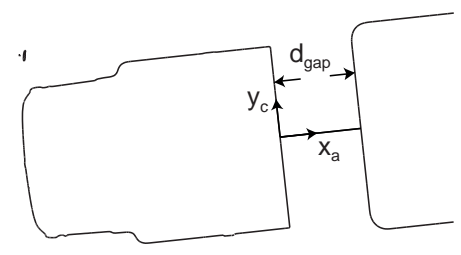

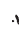

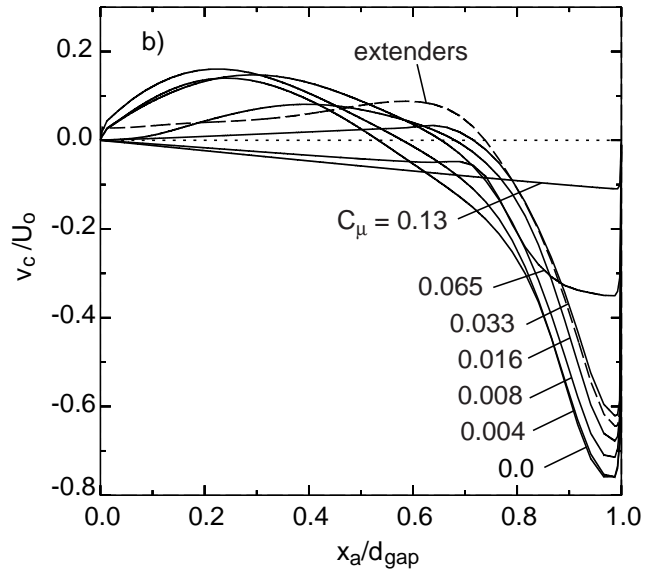

Fig. 10 a) Horizontal cross-section at the mid-height of the tractor-trailer gap. b) Non-dimensional cross-stream velocity through the gap as function of the bleeding coefficient.

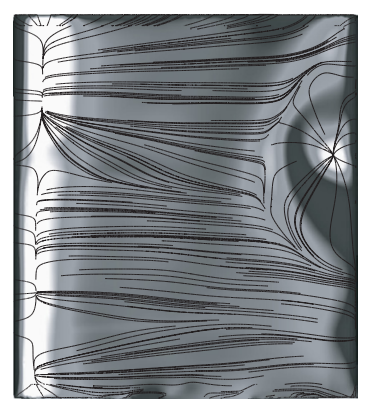

a)

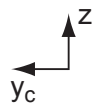

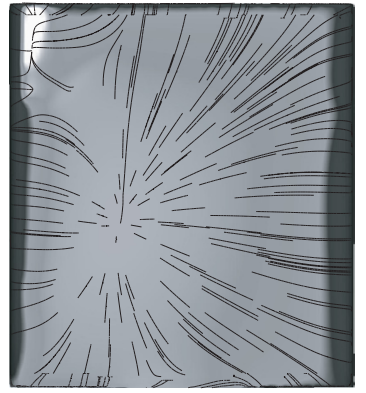

b)

$C_{p}$

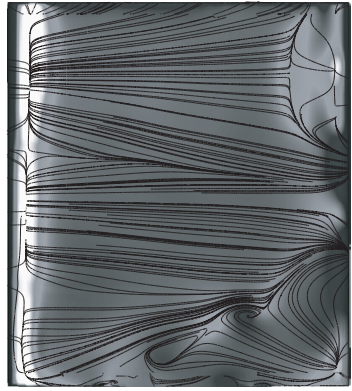

c)

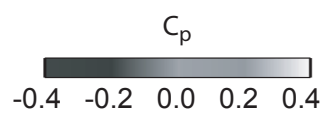

Fig. 11 Pressure coefficient and surface streamlines on the trailer frontal face for $C \mu$ equal to a) 0.0 and b) 0.13 and for c) side extenders.

tractor base and trailer frontal face, which is the mechanism responsible for the drag reduction.

The purpose in selecting tractor base bleeding as a drag reduction concept is to alleviate the maintenance and operational issues that often arise with conventional side extenders. It is evident through the results of this investigation that tractor base bleeding may, in fact, offer an alternative means to side extenders in reducing tractor-trailer gap drag. However, the base bleeding concept is not without its own 

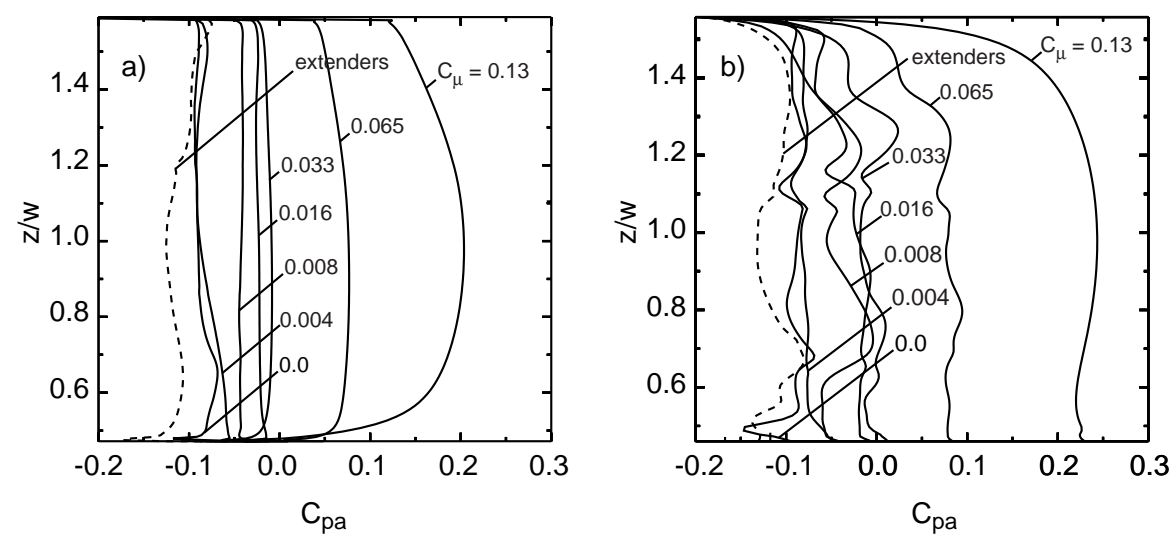

Fig. 12 Pressure coefficient in the body-axis direction on the a) tractor base and b) trailer frontal face centerlines as a function of the bleeding coefficient.

Fig. 13 Average pressure coefficient in the body-axis direction over the tractor base and trailer frontal surface and base in the body-axis direction as a function of the bleeding coefficient.

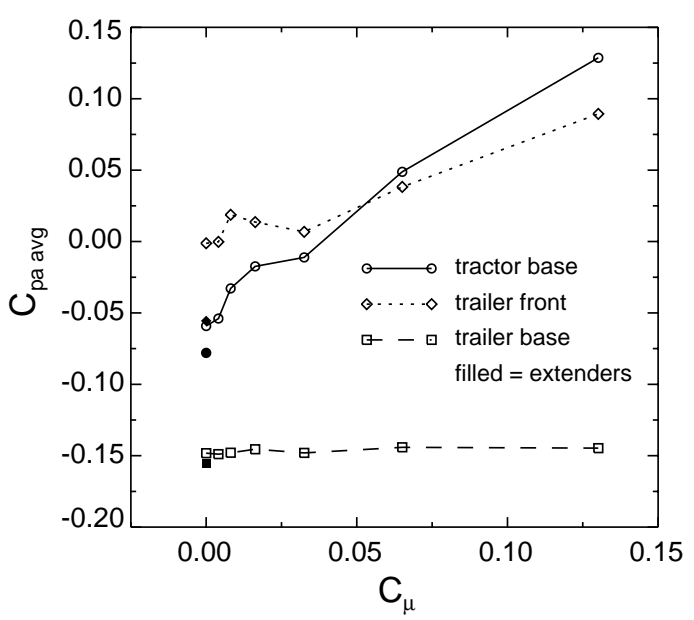

set of concerns and a number of questions remain unanswered regarding its feasibility, some of which are: how much power is required to produce the bleeding flow? Would it be more efficient to utilize solar energy or energy stored from regenerative braking to produce the bleeding flow? Can a base bleeding system be designed robustly enough so as to require a minimum level of maintenance? Under normal operating conditions, does tractor base bleeding provide a net reduction in fuel usage? Lastly, how much would a bleeding system add to the price of a tractor? Obviously, we are not in the position to answer these important questions at the present time. However, if these questions can be adequately and cost effectively addressed, tractor base bleeding may, in the future, offer commercial fleets a viable drag reduction alternative. 


\section{Acknowledgments}

The authors would like to thank Dennis Acosta and Kurt Long of NASA Ames Research Center for their assistance in performing the wind tunnel measurements.

This work performed under the auspices of the U.S. Department of Energy by Lawrence Livermore National Laboratory under Contract DE-AC52-07NA27344.

\section{References}

1. Arcas, D.R., Redekopp, L.G.: Aspects of wake vortex control through base blowing/suction. Phys. Fluids 16(2), 452-456 (2004)

2. Axon, L., Garry, K.P., Howell, J.: An evaluation of CFD for modelling the flow around stationary and rotating isolated wheels. SAE 980032, SAE International Congress and Exhibition, Detroit, Michigan, February 23-26 (1998)

3. Bearman, P.W.: The effect of base bleed on the flow behind a two-dimensional model with a blunt trailing edge. Aero. Quart. 18, 207-224 (1967)

4. BDAS 9.3 Software Package, Aerospace Computing, Inc. (2006)

5. Cooper, K.R.: Truck aerodynamics reborn lessons from the past. SAE 2003-01-3376, SAE International Truck and Bus Meeting and Exhibition, Fort Worth, Texas, November 10-12 (2003)

6. Cooper, K.R.: Commercial vehicle aerodynamic drag reduction: historical perspective as a guide. In: McCallen, R.C., Browand, F., Ross, J. (eds.) The Aerodynamics of Heavy Vehicles: Trucks, Buses, and Trains, pp. 9-28. Springer, Heidelberg (2004)

7. Gill, R.: Freightliner, LLC, private communication (2007)

8. Freightliner, http://www.freightlinertrucks.com/trucks/find-by-model/columbia/(2007)

9. Hammache, M., Browand, F.: On the aerodynamics of tractor-trailers. In: McCallen, R.C., Browand, F., Ross, J. (eds.) The Aerodynamics of Heavy Vehicles: Trucks, Buses, and Trains, pp. 185-205. Springer, Heidelberg (2004)

10. Hammond, D.A., Redekopp, L.G.: Global dynamics of symmetric and asymmetric wakes. JFM 331, 231-260 (1997)

11. Huerre. P., Monkewitz, P.A.: Local and global instabilities in spatially developing flows. Ann. Rev. Fluid Mech. 22, 473-537 (1990)

12. Ingram, K.C.: The wind-averaged drag coefficient applied to heavy goods vehicles. Transport and Road Research Laboratory Supplementary Report 392 (1978)

13. Kays, W.M., Crawford, M.E.: Convective heat and mass transfer. McGraw-Hill, Inc., New York (1993)

14. Koch, W.: Organized structures in wakes and jets-an aerodynamic resonance phenomenon? In: Bradbury, L.J.S., Durst, F., Launder, B.E., Schmidt, F.W., Whitelaw, J.H. (eds.) Turbulent Shear Flows 4, Springer (1983)

15. Koch, W.: Local instability characteristics and frequency determination of self-excited wake flows. J. Sound Vib. 99, 53-83 (1985)

16. Leal, L.G., Acrivos, A.: The effect of base bleed on the steady separated flow past bluff bodies. JFM 39(4), 735-752 (1969)

17. Leuschen, J., Cooper, K.: Full-scale wind tunnel tests of production and prototype, secondgeneration aerodynamic drag-reducing devices for tractor-trailers. SAE Paper 06CV-222, (2006)

18. MacAllister, L.C.: The aerodynamic properties and related dispersion characteristics of a hemispherical-base shell, 90-mm, HE, T91, with and without tracer element. BRL Memo. Report 990, Aberdeen Proving Ground, MD (1956) 
19. Menter, F.R.: Zonal two equation k- $\omega$ turbulence models for aerodynamic flows. Paper No. AIAA 93-2906, Proc. $24^{\text {th }}$ Fluid Dynamics Conf., Orlando, Florida, USA, 6-9 July (1993)

20. Michel, G.W., Kost, F.H.: The effect of coolant flow on the efficiency of a transonic HP turbine profile suitable for a small engine. ASME 82-GT-86 (1982)

21. Pointer, W.D.: Evaluation of commercial CFD code capabilities for prediction of heavy vehicle drag coefficients. Paper No. AIAA-2004-2254, $34^{\text {th }}$ AIAA Fluid Dynamics Conference and Exhibit, Portland, Oregon, 28 June-1 July (2004)

22. SAE wind tunnel test procedure for trucks and buses. SAE J1252, SAE Recommended Practice (1979)

23. Schumm, M., Berger, E., Monkewitz, P.A.: Self-excited oscillations in the wake of twodimensional bluff bodies and their control. JFM 271, 17-53 (1994)

24. Sevilla, A., Martínez-Bazán, C.: Vortex shedding in high Reynolds number axisymmetric bluff-body wakes: local linear instability and global bleed control. Phy. Fluids 16(9), 34603469 (2004)

25. Solid Concepts, Inc., www.solidconcepts.com (2006)

26. STAR-CD v. 3.24-3.26. CD-Adapco Group, www.cd-adapco.com (2007)

27. Turbo Squid, www.turbosquid.com (2006)

28. Wood, C.J.: The effect of base bleed on a periodic wake. J. Roy. Aero. Soc. 68(2), 477-482 (1964)

29. Wood, C.J.: Visualization of an incompressible wake with base bleed. JFM 29(2), 259-272 (1967)

30. Yao, Y.F., Sandham, N.D.: Direct numerical simulation of turbulent trailing-edge flow with base flow control. AIAA J. 40(9), 1708-1716(2002) 